\title{
Gesundheitsförderung und Introvision als Methode der mentalen Selbstregulation
}

\author{
Angelika C. Wagner
}

Introvision ist eine Methode des Selbstmanagements um innere Konflikte aufzulösen, mentale Blockaden zu beenden und dysfunktionale Gewohnheiten zu löschen.

Die Entwicklung der Methode der Introvision ist das Ergebnis eines Langzeitforschungsprogramms an der Universität Hamburg (Wagner 2011) zur Entstehung und Auflösung innerer Konflikte; sie wurde auf der Grundlage umfangreicher empirischer und theoretischer Forschungsarbeiten entwickelt und in unterschiedlichen Anwendungsbereichen erfolgreich erprobt und getestet. Ziel von Training oder Coaching in Introvision ist es, das Individuum zu befähigen, auch und gerade in schwierigen Fällen das eigene Verhalten zu ändern, mentale Blockaden aufzulösen und inneren Stress und Belastung zu verringern. Grundlage der Introvision ist eine bestimmte Form nicht-wertender Aufmerksamkeit, das sogenannte konstatierende aufmerksame Wahrnehmen (KAW). Diese Art der Aufmerksamkeit wird zunächst anhand von vier, eigens dafür entwickelten Übungen über einen längeren Zeitraum geübt. Bei der Introvision wird diese Form der Aufmerksamkeit angewandt, um gezielt einen akuten Konflikt aufzulösen und dysfunktionale Gewohnheiten zu beenden.

Die Durchführung der Introvision umfasst zwei Phasen. Ziel der Phase 1 ist es, den Kern des jeweiligen Konflikts oder der mentalen Blockade zu finden; Grundlage dafür ist die Theorie subjektiver Imperative. Diese beruht auf der Annahme, dass emotionale Konflikte, mentale Blockaden und dysfunktionale Gewohnheiten das Ergebnis unaufgelöster Diskrepanzen im Zusammenhang mit subjektiven Imperativen (z. B. „Ich muss immer perfekt sein!“") sind. In Phase 1 geht es darum, die dem Konflikt zugrundeliegende Subkognition zu finden (z. B. „Es kann sein, dass ich nicht perfekt bin“). In Phase 2 geht es dann darum, diese Subkognition ein Weilchen lang - und ggf. auch wiederholt - aufmerksam konstatierend wahrzunehmen, samt den dazu gehörigen unangenehmen Bildern, Gefühlen oder Tönen. Ziel ist es, auf diese Weise die automatische Koppelung dieser Subkognition mit erhöhter Erregung, Anspannung und Hemmung dauerhaft zu löschen.

Online publiziert: 09.11 .2012

(C) Springer Fachmedien Wiesbaden 2012

Prof. Dr. A. C. Wagner, Ph.D. ( $ه)$

Von-Melle-Park 8, Raum 109, 20146 Hamburg, Deutschland

E-Mail: angelika.wagner@uni-hamburg.de 
In diesem Themenheft geht es um die Frage, was die Anwendung von Introvision zur Förderung von Gesundheit beitragen kann.

Gesundheitsförderung umfasst nach Tannahill (Downie et al. 1996, vgl. auch Hurrelmann 2010) drei einander überlappende Interventionsbereiche: Gesundheitsaufklärung und Gesundheitserziehung, Prävention und Gesundheitsschutz. In allen drei Bereichen spielt die Fähigkeit zur mentalen und emotionalen Selbstregulation eine zentrale Rolle. Dafür gibt es zwei Gründe: Erstens zielen viele Programme und Maßnahmen darauf ab, das Verhalten des einzelnen nachhaltig zu verändern - und das ist oft nicht einfach, wie die Erfahrung zeigt, etwa wenn mentale Blockaden vorliegen. Zweitens spielt bei vielen Maßnahmen die Verringerung von chronischem Stress eine wichtige Rolle - und auch diese ist vielfach nicht leicht erreichbar, insbesondere dann, wenn Ärger oder Angst automatisch aktiviert werden. In diesen beiden Bereichen kann - wie die hier vorliegenden Beiträge zeigen - Introvision einen wesentlichen Beitrag zur Förderung von Gesundheit leisten.

In den folgenden Artikeln werden zum einen verschiedene Programme dargestellt, die auf der Methode der Introvision aufbauen - für Hochleistungssportler und Führungskräfte in der Wirtschaft, für Speditionsmitarbeiter und Pädagoginnen und Pädagogen ebenso wie für Mitarbeiterinnen der Arbeitsagentur und Langzeitarbeitslose. Zum zweiten werden, in vier der fünf Aufsätze, die Ergebnisse empirischer Untersuchungen zur Wirksamkeit der jeweiligen Interventionskonzepte ausführlich vorgestellt.

A. C. Wagner gibt im ersten Beitrag dieses Heftes eine allgemeine Übersicht über die Ergebnisse von fünf quantitativen Interventionsstudien (Wartezeitkontrollgruppendesign) zur Wirksamkeit von Introvision bei verschiedenen Zielgruppen (z. B. Berufstätige, Leistungssportler, weibliche Führungskräfte) und in verschiedenen Anwendungsbereichen (z. B. Sress und Belastung, Tinnitus, chronische Nackenverspannungen, Schwerhörigkeit). Die Ergebnisse belegen, dass die Anwendung von Introvision als Selbstmanagement-Tool zu einer signifikanten und langfristig wirksamen Verringerung von chronischem Stress und Belastung als auch zur Verringerung spezifischer gesundheitsbeeinträchtigender Probleme (Hörfähigkeit, dauerhafte Verringerung von chronischen Verspannungen im Rücken) führt.

B. Buth und N. Pereira Guedes gehen im zweiten Beitrag differenzierter der Frage nach, wie sich dieser Effekt der Verringerung von chronischem Stress durch Introvision theoretisch und empirisch erklären lässt. Zu diesem Zweck nehmen sie eine Re-Analyse der Daten aus ihren beiden Forschungsprojekten zur Anwendung von Introvision bei Tinnitus (Buth) und chronischen Nackenverspannungen (Guedes) vor. Insgesamt zeigte sich, dass die generellen Stress- und Belastungswerte bei den Tinnitusbetroffenen teilweise signifikant höher waren als bei den Personen mit chronischen Nackenverspannungen. Dies ist, so argumentieren sie, v. a. auf unterschiedliche Konfliktumgehungsstrategien zurückzuführen - Dramatisieren (Tinnitus) vs. Bagatellisieren (Nackenverspannungen). Im Übrigen zeigen sie, dass die Anwendung von Introvision dazu führt, die Tendenz zur Chronifizierung (und damit zur langfristigen Verschlimmerung der Problematik) aufzuhalten und z. T. sogar tendentiell umzukehren.

S. Löser stellt im dritten Beitrag eine Pilotstudie zu den Auswirkungen von Coaching in Introvision bei Langzeitarbeitslosen und Mitarbeiterinnen einer Arbeitsagentur im Rahmen eines Gesundheitsförderprogramms vor. Die Teilnehmer waren zum einen 
schwer vermittelbare Langzeitarbeitslose über 50 und zum zweiten Fallmangerinnen. Die Ergebnisse zeigen, dass sowohl die Arbeitslosen als auch die Fallmanagerinnen von dem Programm deutlich profitierten - dies zeigte sich insbesondere in einem verbesserten Umgang mit Stress, in der Verringerung von sozialer Isolation, verbesserter Selbstwahrnehmung und in einer offeneren Zusammenarbeit zwischen Kundinnen und Mitarbeiterinnen der ARGE. Außerdem gelang es anschließend, vier (von 20) Langzeitarbeitslosen eine (sozialversicherungspflichtige) Stelle sowie zwei weiteren eine ehrenamtliche Tätigkeit zu vermitteln.

T. A. Iwers-Stelljes stellt im vierten Beitrag das Modell der introvisionsorientierten Fallreflexion für Pädagoginnen und Pädagogen vor. Dieses von Iwers-Stelljes und R. Luca an der Universität Hamburg gemeinsam entwickelte, vielfach praktisch erprobte und empirisch evaluierte Modell dient dem Ziel der Gesundheitsförderung im Rahmen der Professionalisierung von PädagogInnen. In dem vorliegenden Beitrag wird dieses Modell im Kontext des Salutogenese-Konzepts von Antonovsky ausführlich dargestellt und analysiert. Dabei arbeitet die Verfasserin insbesondere die Bedeutsamkeit des Perspektivwechsels und der introvisionsorientierten Fallanalyse für die Entwicklung von Kohärenz heraus. Was das praktisch bedeutet, wird abschließend anhand eines Fallbeispiels aus der Seminarpraxis erläutert.

B. Wollesen, H. Lex und K. Mattes stellen im fünften Beitrag empirische Ergebnisse einer Interventionsstudie zur Wirksamkeit des an der Universität Hamburg entwickelten BASE-Konzepts zur Prävention von Rückenbeschwerden im Rahmen der betrieblichen Gesundheitsförderung vor. Der multimodale BASE-Ansatz beruht unter anderem auf der der Introvision zugrundeliegenden Theorie der Verhaltensänderung. Ziel des BASE-Programms war es, die Mitarbeiter in einer Spedition in ergonomischerem Bewegungsverhalten bei der manuellen Lastenhandhabung zu schulen. Wie die Ergebnisse zeigen, führte das BASE-Konzept u.a. zu positiven Effekten im Bereich der Motivation und zu einer funktionaleren kognitiven Repräsentation des Bewegungsverhaltens im Langzeitgedächtnis.

Insgesamt befassen sich die verschiedenen Aufsätze in diesem Themenheft mit unterschiedlichen Möglichkeiten, die Introvision erfolgreich als Methode der mentalen Selbstregulation zur Förderung der Gesundheit einzusetzen: zur gezielten Verringerung störungsspezifischer Probleme (z.B. Tinnitus, Schwerhörigkeit, chronische Rückenverspannungen), zur Reduktion von chronischem Stress und Belastung (Hochleistungssportler, Führungskräfte, Arbeitslose) sowie - teilweise auch in Verbindung mit anderen Ansätzen (Fallreflexion, Bewegungsschulung) - zur Primär- und Sekundärprävention der Entwicklung längerfristiger gesundheitlicher Probleme.

\section{Literatur}

Downie, R. S., Tannahill, C., Tannahill, A. (1996). Health promotion: Models and values. Oxford: Oxford University Press.

Hurrelmann, K. (2010). Gesundheitssoziologie. Eine Einführung in sozialwissenschaftliche Theorien von Krankheitsprävention und Gesundheitsförderung (7. Aufl). Weinheim: Juventa.

Wagner, A. C. (2011). Gelassenheit durch Auflösung innerer Konflikte. Mentale Selbstregulation und Introvision (2.,vollst. überarb. und erweiterte Auflage). Stuttgart: Kohlhammer. 